\title{
Editorial \\ Development of Alternative Green Sample Preparation Techniques
}

\author{
Emanuela Gionfriddo \\ Department of Chemistry and Biochemistry, Dr. Nina McClelland Laboratory for Water Chemistry and \\ Environmental Analysis, School of Green Chemistry and Engineering, The University of Toledo, \\ Toledo, OH 43606, USA; Emanuela.Gionfriddo@UToledo.Edu
}

Received: 16 April 2020; Accepted: 8 May 2020; Published: 4 June 2020

Although chemistry disciplines are often regarded by the public as polluting sciences, in the last three decades, the concept of "Green Chemistry" has fueled the development of more sustainable and environmentally friendly chemical processes that are mainly aimed at minimizing the production of toxic laboratory waste, to maximize pollution prevention [1]. Since the establishment of the 12 principles of Green Analytical Chemistry, the analytical chemistry community is striving to apply these principles in the analytical chemistry laboratory, which redefines analytical procedures, with a drastically changed philosophy on analytical method development [2-5]. Among the various steps that constitute the analytical workflow, sample preparation and extraction showed great potential for improvement toward greener approaches, especially for complex matrices, whether for targeted or non-targeted analyses, which present many analytical challenges. Many researchers in the analytical chemistry community have subsequently embraced the challenge and focused their research efforts toward greener and faster sample preparation approaches, guaranteeing minimal consumption of organic solvents, promoting the production of reusable extraction devices, enhancement of analysis throughput through the use of automated systems, use of natural sorptive materials, etc. [6].

The Special Issue of Separations, "Development of Alternative Green Sample Preparation Techniques", aims to provide an update on recent trends in green sample preparation to readers already familiar with the topic and hopefully spark the curiosity and the attention of more analytical chemists toward the importance of this topic.

This Special Issue of Separation collates 11 impressive contributions that describe the state-of-the-art in the development of green extraction technologies, from green materials for microextraction to the development of new sampling devices geometries for enhanced extraction efficiency and analysis throughput.

Seven review articles describe important aspects of green sample preparation:

In terms of green materials for sample preparation, two interesting reviews provide insights on green synthesis of sorbents and use of biomaterials.

Veronica Pino and coworkers at the University of La Laguna (Spain) reviewed the use of Metal-Organic Frameworks (MOFs) as sorbent materials for green sample preparation. The authors survey the characteristics of these materials, giving particular emphasis to potential toxicity issues of neat MOFs and alterative synthetic routes, to ensure green approaches in their preparation [7].

As an alternative to synthetic sorbents, and to further green sample preparation techniques, the use of biopolymers, obtained from renewable natural sources, has attracted the interest of many researchers in the field of sample preparation. Eduardo Carasek and coworkers at the Universidade Federal de Santa Catarina (Brazil) described how various biosorbents can be used as extraction phases for different extraction techniques and discussed several applications to environmental, food, and biofluids analysis. Moreover, the authors describe the use of alternative environmentally-friendly extraction phases, such as Ionic Liquids and SupraMolecular Solvents [8]. 
Two reviews in this Special Issue discuss the development of the geometries of microextraction devices for enhanced sample throughput and extraction efficiency:

The first review article in the literature exclusively dedicated to the development and application of Arrow SolidPhase Microextraction (SPME) was written by Jason S. Herrington, German A. Gómez-Ríos and coworkers at Restek Corporation (USA). This article reports the development of a novel SPME geometry, Arrow SPME, which guarantees enhanced mechanical robustness, compared to classical SPME fibers, with improved extraction efficiency. Practical aspects of the use of Arrow SPME were described, together with several applications in environmental, food, cannabis, and forensic analysis. Moreover, novel interfaces for direct coupling of Arrow SPME to mass spectrometry and recent developments in coating materials were discussed [9].

Moreover, my research group at The University of Toledo (USA) provided a review article that focused on the development and applications of thin-film microextraction devices for thermal desorption. In this article, we provided a comprehensive discussion on the development of TF-SPME for thermal desorption. Practical tips for method development and optimization of Thin-Film-SolidPhase Microextraction-ThermalDesoptionUnit-Gas Chromatography/Mass Spectrometry (TF-SPME-TDU-GC/MS) protocols were discussed. Additional detailed outlook on the current progress of TF-SPME development and its future has also been discussed, with emphasis on its applications to environmental, food, and fragrance analysis [10].

The importance of green extraction techniques as applied to clinical/bio-analysis was reviewed in two contributions in this Special Issue:

The applicability of sampling techniques in clinical settings requires the use of non-invasive protocols that pose no harm to living systems, while providing a high degree of pre-concentration and specificity for the analytes of interest. In this context, Ezel Boyaci and coworkers at the Middle East Technical University (Turkey) wrote a review on new approaches to non-invasive biological surface sampling and discussed recent developments in non-invasive in vivo and in situ sampling methods from biological surfaces. Directions for the development of future technology and potential areas of applications, such as clinical, bioanalytical, and doping analyses, were also discussed [11].

Katarzyna Madej and Wojciech Piekoszewski from Jagiellonian University (Poland), provided an interesting overview of the most commonly used microextraction techniques for analysis of biofluids. Considering the complexity of biofluids, modern clinical and forensic toxicological analysis seeks sample preparation methods characterized by high selectivity and enrichment capability, and easy automation and miniaturization, with minimal sample size requirement. These unique features were well documented and described in this review, focusing on microextraction approaches, such as liquid-phase techniques (e.g., single-drop microextraction, SDME; dispersive liquid-liquid microextraction, DLLME; hollow-fiber liquid-phase microextraction, HF-LPME) and sorbent-based extraction techniques (solid-phase microextraction, SPME; microextraction in packed syringes, MEPS; disposable pipette tip extraction, DPX; stir bar sorption extraction, SBSE) [12].

Finally, among the review articles is an interesting contribution on the evolution of green-sample preparation strategies for metal extraction from Jon R. Kirchhoff's research group at the University of Toledo (USA). Extraction of metal analysis is a topic rarely discussed in terms of microextraction techniques and green sampling procedures. The review article thus offers readers a clearly defined viewpoint on various extraction strategies that minimize the use of organic solvents, such as the application of micro-methodology to minimize waste with reduced costs, improved safety, and the utilization of benign or reusable materials for extraction of metal ions from environmental samples by solvent- and sorbent-based extraction techniques [13].

In this Special Issue, four research papers have been collated from experts in the field of analytical chemistry:

At the University of Campinas (Brazil), Leandro W. Hantao's group developed a HeadSpace-SolidPhase Microextraction comprehensive two-dimensional gas chromatographic/Mass Spectrometry (HS-SPME-GCxGC/MS) method to establish the contribution of Brazilian Ale 02 yeast 
strain to the aroma profile of beer compared with the traditional Nottingham yeast. The use of HS-SPME enabled sampling of the beer aroma and the introduction of the extracted analytes into the comprehensive two-dimensional gas chromatographic system, without the use of organic solvents. Critical in this study was also the application of a multiway principal component analysis approach for data processing to distinguish beer samples based on yeast strain [14].

José Manuel F. Nogueira and coworkers at the University of Lisbon (Portugal), developed a new generation of bar adsorptive microextraction devices $(\mathrm{BA} \mu \mathrm{E})$ that, combined with micro-liquid desorption, followed by high-performance liquid chromatography-diode array detection $(\mathrm{BA} \mu \mathrm{E}-\mu \mathrm{LD} / \mathrm{HPLC}-\mathrm{DAD})$, enabled the determination of two very polar ultraviolet (UV) filters (2-phenylbenzimidazole-5-sulfonic acid (PBS) and 5-benzoyl-4-hydroxy-2-methoxybenzenesulfonic acid (BZ4)) in aqueous media. This contribution showed an innovative analytical cycle that includes the use of disposable devices, making $\mathrm{BA} \mu \mathrm{E}$ a user-friendly and suitable approach for routine work and a remarkable analytical alternative for trace analysis of priority compounds in real sample matrices [15].

Another contributing work to the green extraction procedure can be seen from the work by Paul D. Roach and coworkers, as they explored the use of water (non-toxic solvent) for effective extraction of trypsin inhibitors from defatted Gac (Momordica cochinchinensis Spreng) seeds, to produce trypsin-inhibitor-enriched freeze-dried powder. The optimization of the extraction procedure resulted in high-quality powder in terms of its highly specific trypsin inhibitor activity (TIA) and physical properties [16].

An additional important aspect of green extraction technology is, without doubt, the ability of miniaturization and automation. Considering these factors, Marc A. Althoff and coworkers developed a Smart-SolidPhase Extraction (Smart-SPE) protocol for analysis of chemical warfare agents. The innovation demonstrated in the ability to use fully automated analytical workflows that included several steps, such as sample vortexing, liquid-liquid extraction, and $\mu$-SPE. Such fully automated analytical workflows avoid errors prone to off-line protocols and can help to minimize health risks for lab personnel when toxic substances may be analyzed [17].

Serving as Guest Editor of this Special Issue has been a very exciting experience. I would like to express my deepest gratitude to all the authors for their brilliant contributions and invite the readers of Separations to take full advantage of all the important information that this Special Issue provides, hoping that many of these strategies will be broadly applied in many analytical chemistry laboratories.

Funding: This research was funded by The University of Toledo.

Conflicts of Interest: The authors declare no conflict of interest.

\section{References}

1. Anastas, P.T.; Warner, J.C. Green Chemistry: Theory and Practice; Oxford University Press: New York, NY, USA, 1998; Available online: https://books.google.com/books/about/Green_Chemistry.html?id=SrO8QgAACAAJ (accessed on 17 July 2019).

2. Tobiszewski, M.; Mechlińska, A.; Namieśnik, J. Green analytical chemistry-Theory and practice. Chem. Soc. Rev. 2010, 39, 2869-2878. [CrossRef]

3. Gałuszka, A.; Migaszewski, Z.; Namieśnik, J. The 12 principles of green analytical chemistry and the SIGNIFICANCE mnemonic of green analytical practices. TrAC-Trends Anal. Chem. 2013, 50, 78-84. [CrossRef]

4. Armenta, S.; Garrigues, S.; de la Guardia, M. The role of green extraction techniques in Green Analytical Chemistry. TrAC-Trends Anal. Chem. 2015, 71, 2-8. [CrossRef]

5. Armenta, S.; Garrigues, S.; de la Guardia, M. Green Analytical Chemistry. TrAC—Trends Anal. Chem. 2008, 27, 497-511. [CrossRef]

6. Armenta, S.; Garrigues, S.; Esteve-Turrillas, F.A.; de la Guardia, M. Green extraction techniques in green analytical chemistry. TrAC-Trends Anal. Chem. 2019, 116, 248-253. [CrossRef]

7. Rocío-Bautista, P.; Taima-Mancera, I.; Pasán, J.; Pino, V. Metal-organic frameworks in green analytical chemistry. Separations 2019, 6, 33. [CrossRef] 
8. Carasek, E.; Bernardi, G.; Carmo, S.N.D.; Vieira, C.M.S. Alternative green extraction phases applied to microextraction techniques for organic compound determination. Separations 2019, 6, 35. [CrossRef]

9. Herrington, J.S.; Gómez-Ríos, G.A.; Myers, C.; Stidsen, G.; Bell, D.S. Hunting molecules in complex matrices with spme arrows: A review. Separations 2020, 7, 12. [CrossRef]

10. Emmons, R.V.; Tajali, R.; Gionfriddo, E. Development, optimization and applications of thin film solid phase microextraction (TF-SPME) devices for thermal desorption: A comprehensive review. Separations 2019, 6, 39. [CrossRef]

11. Nalbant, A.A.; Boyac1, E. Advancements in non-invasive biological surface sampling and emerging applications. Separations 2019, 6, 52. [CrossRef]

12. Madej, K.; Piekoszewski, W. Modern Approaches to Preparation of Body Fluids for Determination of Bioactive Compounds. Separations 2019, 6, 53. [CrossRef]

13. Sunder, G.S.S.; Adhikari, S.; Rohanifar, A.; Poudel, A.; Kirchhoff, J.R. Evolution of Environmentally Friendly Strategies for Metal Extraction. Separations 2020, 7, 4. [CrossRef]

14. Paiva, A.C.; Oliveira, D.S.; Hantao, L.W. A Bottom-Up Approach for Data Mining in Bioaromatization of Beers Using Flow-Modulated Comprehensive Two-Dimensional Gas Chromatography/Mass Spectrometry. Separations 2019, 6, 46. [CrossRef]

15. Ide, A.H.; Nogueira, J.M.F. Determination of Hydrophilic UV Filters in Real Matrices Using New-Generation Bar Adsorptive Microextraction Devices. Separations 2019, 6, 45. [CrossRef]

16. Le, A.V.; Parks, S.E.; Nguyen, M.H.; Roach, P.D. Optimised Extraction of Trypsin Inhibitors from Defatted Gac (Momordica cochinchinensis Spreng) Seeds for Production of a Trypsin Inhibitor-Enriched Freeze Dried Powder. Separations 2019, 6, 8. [CrossRef]

17. Althoff, M.A.; Bertsch, A.; Metzulat, M. Automation of $\mu$-SPE (Smart-SPE) and Liquid-Liquid Extraction Applied for the Analysis of Chemical Warfare Agents. Separations 2019, 6, 49. [CrossRef]

(C) 2020 by the author. Licensee MDPI, Basel, Switzerland. This article is an open access article distributed under the terms and conditions of the Creative Commons Attribution (CC BY) license (http://creativecommons.org/licenses/by/4.0/). 